\title{
mHealth para mejorar la higiene oral de niños. Revisión de literatura.
}

\section{mHealth to improve oral health in children. Literature review.}

\author{
M. J. Pacheco-Vergara*, R. A. Cartes-Velásquez**
}

\section{RESUMEN}

Las intervenciones educativas en salud oral han cambiado a través del tiempo, partiendo desde la simple entrega de información se ha avanzado hacia programas que utilizan estrategias psicológicas para motivar el cambio de conducta. La incorporación de internet en smartphones junto con el amplio uso de ellos entrega la oportunidad para realizar intervenciones innovadoras en salud con mHealth (salud móvil) para mejorar la salud y calidad de vida a nivel mundial. El uso de nuevas tecnologías está presente en el día a día de los niños, lo que entrega la oportunidad de promocionar la salud oral de una forma didáctica a los nativos digitales. El objetivo de la presente revisión de literatura es describir los resultados de la evidencia reciente del uso de aplicaciones móviles o Apps para mejorar la higiene oral en niños. La tecnología con smartphones ha crecido a un ritmo acelerado junto con el desarrollo de Apps, sin embargo, la investigación no ha llevado el ritmo. Se necesitan estudios estandarizados y específicos para cada población, especialmente en niños, antes de aprobar una App y dejarla disponible para descargar. De esta manera se podrán generar cambios efectivos a largo plazo respecto a los hábitos de salud oral de niños y adultos.

PALABRAS CLAVE: mHealth, higiene oral, niños, Smartphone.

\section{ABSTRACT}

Educational interventions in oral health have changed over time, starting from the simple delivery of information, progress has been made towards programs that use psychological strategies to motivate behavior change. The incorporation of the internet in smartphones along with the wide use of them provides the opportunity to carry out innovative health interventions with mHealth (mobile health) to improve health and quality of life worldwide. The use of new technologies is present in the daily lives of children, which gives the opportunity to promote oral health in a didactic way to digital natives. The aim of this literature review is to describe the results of recent evidence of the use of mobile applications or Apps to improve oral hygiene in children. Technology with smartphones has grown at an accelerated pace along with the development of Apps, however, research has not kept pace. Standardized and specific studies are needed for each population, especially in children, before approving an App and making it available for download. In this way, effective long-term changes can be generated regarding the oral health habits of children and adults.

KEY WORDS: mHealth, higiene oral, niños, Smartphone.

Fecha de recepción: 1 de septiembre de 2019

Fecha de aceptación: 15 de septiembre de 2019

M. J. Pacheco-Vergara, R. A. Cartes-Velásquez. mHealth para mejorar la higiene oral de niños. Revisión de literatura. Av Odontoestomatol. 2020; 36, (1): 27-34.

* $\quad$ Fundación Kimntrum, Concepción, Chile.

* * Facultad de Odontología, Universidad Andrés Bello, Sede Concepción, Chile.

Facultad de Ciencias de las Salud, Universidad Autónoma de Chile, Temuco, Chile. 


\section{INTRODUCCIÓN}

La caries dental en niños es la enfermedad crónica más prevalente, por ello se considera una crisis de salud pública a nivel mundial. ${ }^{(1,2,3,4)}$ En Latinoamérica y el Caribe, más de $50 \%$ de los preescolares de 5-6 años presentan caries dental.(3) Una buena higiene oral es fundamental para la prevención de enfermedades orales, ${ }^{(5,6)}$ siendo clave el cepillado dental dos veces al día para prevenir caries. ${ }^{(7)}$ Una higiene oral deficiente en niños genera múltiples problemas incluyendo ausentismo escolar, deficiente ingesta nutricional y restricción en las actividades diarias que conduce a un menor desarrollo cognitivo y crecimiento. ${ }^{(1,6,8)}$ Esto trae repercusiones en la autoestima, estética, fonación y masticación del niño. (3) La caries es un problema donde influyen factores socioeconómicos $^{(9)}$ e impacta de forma significativa la calidad de vida, siendo fundamental para el bienestar y salud general. ${ }^{(5,6)}$

Las intervenciones educativas en salud oral han cambiado a través del tiempo, partiendo desde la simple entrega de información se ha avanzado hacia programas que utilizan estrategias psicológicas para motivar el cambio de conducta. ${ }^{(2,10)}$ Educar a los niños acerca de su salud es un punto estratégico ya que a través de ellos se puede llegar a sus familias y la comunidad, permitiendo cambios de actitud y comportamientos en una etapa temprana. ${ }^{(10)}$ Durante los años preescolares se forman los hábitos, por ello es importante promocionar una buena higiene oral en ese momento. ${ }^{(3)}$ Además, se ha demostrado que los niños pueden transmitir conocimientos a sus padres. (11) Se debe motivar a los niños para que sean ellos los que se responsabilicen del cepillado dental, ${ }^{\left({ }^{8}\right)}$ ya que la evidencia sugiere que desde una edad temprana los niños tienen cierto papel en sus hábitos de higiene oral ${ }^{(11)}$ y terminando el día los padres tienden a dejar pasar las peleas del cepillado de dientes. ${ }^{\left({ }^{(8)}\right.}$

Evidencia reciente muestra que mediante el uso de tecnología se ha mejorado el acceso a la información y cuidados en salud de una manera más costo-efectiva, permitiendo la promoción de la salud en todo momento y lugar. ${ }^{(12,13,14)}$ La incorporación de internet en smartphones junto con el amplio uso de ellos entrega la oportunidad para realizar intervenciones innovadoras en salud con mHealth (salud móvil) para mejorar la salud y calidad de vida a nivel mundial. ${ }^{(12,13,15)} \mathrm{El}$ uso de nuevas tecnologías está presente en el día a día de los niños, lo que entrega la oportunidad de promocionar la salud oral de una forma didáctica a los nativos digitales. ${ }^{(3)}$ El objetivo de la presente revisión de literatura es describir los resultados de la evidencia reciente del uso de aplicaciones móviles o Apps para mejorar la higiene oral en niños.

\section{Uso DE TECNOLOGía PARA LA PROMOCIÓN DE LA SALUD}

En los últimos años, el uso de celulares se ha expandido por todo el mundo, abarcando el $90 \%$ de la población mundial, con casi $80 \%$ de cobertura en zonas rurales. ${ }^{(12)}$ Los celulares son portados prácticamente en todo momento, manteniéndolos al alcance de la mano gran parte del tiempo y se han transformado en un objeto apreciado para las personas, creando un fuerte apego emocional a ellos. ${ }^{(13,16)}$. Este apego es más notorio aún en niños que han crecido rodeados de tecnología, lo que puede beneficiar la promoción de salud, ${ }^{(3,16)}$ ya que son influenciables por la publicidad y medios. ${ }^{(11)}$ Los avances tecnológicos en smartphones y Apps han influenciado el acceso a la información y métodos de comunicación paciente-profesional en salud. ${ }^{(15)}$ La versatilidad de los smartphones permite la entrega de información visual, textual y con videos, además de permitir personalizar la información para enviar recordatorios y monitorizar el progreso individual. ${ }^{(4,17)}$

El uso de Apps, mensajes de texto e internet han demostrado que permiten influenciar comportamientos de salud en la población para controlar enfermedades crónicas, ${ }^{(2,12,13)}$ mejorando el control de factores de riesgo, la adherencia a tratamientos, ${ }^{(18)}$ y compromiso de los pacientes. ${ }^{(19)}$ Usando Apps es posible mejorar la salud de la población disminuyendo los $\operatorname{costos}^{(12,20,21)}$ incorporando técnicas para cambio de conducta. ${ }^{(22)}$ Esto también incluye a la población sana para promocionar hábitos saludables y prevenir enfermedades. ${ }^{(14)}$ mHealth ha demostrado ser una herramienta eficaz en poblaciones marginadas, de bajo nivel socioeconómico ${ }^{(2)}$ y países subdesarrollados, entregando mensajes adaptados a las condiciones de cada grupo, equiparando el uso entre niveles socioeconómicos. ${ }^{(12)}$

Con el incremento de internet en los hogares, el tiempo de uso de medios tecnológicos en niños se promedia a 8 horas al día, lo que puede utilizarse para influenciar su salud entregando mensajes personalizados. El uso de Apps presenta las ventajas de usarlas en tablets, por lo que niños que no tengan acceso a celulares pueden tener acceso a las Apps con tablets en sus casas, ${ }^{(16)}$ y pueden ser un motiva- 
dor externo para ellos al incluir tecnología y medios sociales. ${ }^{\left({ }^{8}\right)}$ Otras ventajas de mHealth son la facilidad de acceso a la información, posibilidad de agregar recordatorios y motivar a los pacientes, hacer seguimiento del progreso y lograr mayor compromiso del paciente. Por otro lado, las Apps presentan desventajas, ya que con su rápido incremento existe falta de regulación, por lo que las apps existentes pueden contener información errónea. ${ }^{(19)}$ A pesar de lo anterior, los resultados de estudios obtenidos para promoción de salud dental con medios tecnológicos han sido positivos, utilizando principalmente las estrategias de retroalimentación y seguimiento para motivar a la población. ${ }^{(23)}$

\section{Características de las Apps de mHealth efectivas}

Para crear una App efectiva en mHealth, se deben considerar diversos componentes de la población, accesibilidad, acceso a internet y uso de dispositivos. Se debe comenzar por verificar la penetración de dispositivos móviles en la población blanco, seguido por la capacidad de instalar Apps en dichos dispositivos. Luego se debe evaluar el acceso a internet con banda ancha móvil desde los smartphones ya que las Apps generalmente necesitan conexión a internet rápida para el desarrollo de sus funciones. (24) En lugares con poco acceso a internet, o acceso intermitente, será mejor desarrollar una App que pueda utilizarse sin internet una vez descargada. ${ }^{(18)}$ Por último, se sugiere evaluar el uso de dispositivos personales por los individuos, lo que permite personalizar el contenido de la App a cada usuario. ${ }^{(24)}$

Stephan et al. (2017) proponen cinco pasos para crear una App de mHealth con base científica, dirigida a poblaciones de países subdesarrollados o en vías de desarrollo. Primero, se debe identificar y caracterizar a la población de interés, el problema a tratar y el objetivo que se espera lograr. Segundo, se debe realizar una revisión de literatura para basarse en la mejor evidencia científica y datos de la población de interés, seguido por la generación de conocimiento a partir de la información obtenida. En este paso se diseña la App para adaptar la información recopilada al nivel de educación, lenguaje y cultura de los usuarios, personalizando los mensajes. La App debe ser fácil de navegar, presentar información clave y concisa, pero que entregue más información del tema si el usuario lo desea, y debe tener un diseño atractivo. Es importante asegurar la protección de la información de los usuarios. Por último, evaluar su eficacia y uso con un estudio piloto, evaluación de los resultados obtenidos a corto y largo plazo, y retroalimentación permanente desde la misma App. ${ }^{(18)}$

Otros factores que se deben considerar para asegurar el uso de la App son factores psicológicos y sociodemográficos. Se ha demostrado que las mujeres y los más jóvenes son los que más utilizan Apps. ${ }^{(22)}$ Es importante considerar la cultura y tipo de motivación de la población de interés, ya que son factores que influenciará si se obtienen cambios de conducta utilizando encuadres de ganancia o de pérdida. Personas de culturas que busquen el éxito responderán mejor con mensajes de ganancia en salud, mientras que personas de culturas que tienden a evitar pérdidas van a responder a mensajes que destaquen pérdidas de salud. ${ }^{(25)}$

Para lograr cambios positivos en salud con Apps de mHealth, es necesario que los usuarios mantengan el interés para utilizar la App por tiempos prolongados. Las Apps bien evaluadas son aquellas intuitivas de usar, donde se permite compartir los datos, y que ofrezcan un método más rápido y eficiente para cuidar su salud que las otras alternativas existentes. ${ }^{(20)}$ Gamification o ludificación es un término utilizado para agregar diversión a contextos fuera de los juegos. Se enfoca en agregar mecanismos del juego para influenciar el comportamiento y motivar al público en tareas del día a día. ${ }^{(26,27)}$ El objetivo es generar una experiencia asimilable a un juego, entregando premios, retroalimentación, creando competencia e interacción entre usuarios con la incorporación de redes sociales, entre otros. Al aplicar gamification, las tareas que antes eran consideradas aburridas se vuelven entretenidas y captan al público para comprometerse con las tareas a largo plazo. Este enfoque se ha utilizado en salud para mejorar el bienestar general con la promoción de estilos de vida saludables ${ }^{(26)}$ y mejorar el autocontrol de enfermedades. ${ }^{(11)}$

Los beneficios reportados con la ludificación en mHealth son aumento del compromiso de intervenciones en salud, mejor autocontrol de la condición de salud y adherencia al tratamiento, aumento de la satisfacción y el autoestima, mejor estado emocional y motivación, se refuerza el cambio de conductas saludables, aumenta las competencias sociales y transforma las actividades "aburridas" en divertidas y entendibles. Al aumentar la motivación intrínseca, la autoestima y la satisfacción, se logran cambios 
positivos en el comportamiento de los usuarios que benefician su bienestar y salud general.(26) Estudios han demostrado que el diseño de los elementos del juego son los que mejoran la motivación al inducir las necesidades psicológicas de competencia, autonomía y deseos de pertenecer a un grupo. La retroalimentación genera competencia y la percepción de realizar una tarea significativa. ${ }^{(20,27)}$. Por otro lado, la experiencia de pertenecer a un grupo puede ser creada con la presencia compañeros en el juego, la creación de historias significativas, el uso de ava$\operatorname{tars}^{(27)}$ y conectándose a otros medios sociales ${ }^{(20)}$. Otros factores que influyen en la motivación con gamification son la calidad y estética del juego, por lo que se les debe dar importancia. ${ }^{(27)}$

Se ha demostrado que los mensajes personalizados son más efectivos para realizar cambios positivos de conducta en salud, respecto a mensajes no personalizados. ${ }^{(1,18)}$ Personalizar un mensaje implica conocer las características de la población de interés, sus actitudes, motivaciones, comportamientos y barreras, para que la información entregada sea relevante. ${ }^{(1,8)}$ Este tipo de mensajes se lee, entiende y recuerda mejor. Además, son encontrados más interesantes, obteniendo mayor éxito para influenciar el comportamiento, motivando la adopción de comportamientos saludables. ${ }^{(1,8)}$ Con el uso de dispositivos móviles, es posible entregar mensajes personalizados a cada persona de acuerdo a sus características. ${ }^{(12)} \mathrm{El}$ uso de imágenes para enfatizar la información entregada mejora el aprendizaje, comprensión del mensaje, se queda en la memoria a largo plazo y permite desencadenar emociones. ${ }^{(18)}$ También se mejora la motivación al asociar una imagen con el estado actual de salud del individuo. ${ }^{(14)}$

Se ha sugerido que intervienen dos procesos en la toma de decisiones, un proceso cognitivo emocional y otro proceso analítico o racional. Para individualizar los mensajes, se debe abarcar el proceso racional, lo que entrega resultados positivos en hábitos de salud a largo plazo y disminuye los costos en salud. Las campañas de salud que utilizan el proceso emocional entregan información de "talla única" para todos sin lograr motivar a la población y no se obtienen los resultados deseados. ${ }^{(28)}$

\section{MHEALTH PARA PROMOCIONAR LA SALUD ORAL}

Para que los pacientes realicen cambios significativos en sus hábitos de higiene oral es necesario acompañar las instrucciones orales con ilustraciones visuales y escritas. Mediante mHealth es posible entregar instrucciones con ilustraciones, aumentando la adherencia a los hábitos de higiene al motivar más a los pacientes, disminuyendo la inflamación gingival y cantidad de placa. ${ }^{(13)}$ Estrategias como la creación de juegos educativos con animación y audio dentro de una App móvil pueden ser utilizados para educar a preescolares acerca de su salud oral. ${ }^{(3)}$

En niños, la falta de comprensión en sus decisiones conduce a la necesidad de profundizar en sus creencias, motivaciones y prácticas para generar mensajes personalizados y lograr mejorar su salud oral, ${ }^{(1,8)}$ sin olvidar que procesan el mensaje distinto a los adultos. ${ }^{(1)}$ Utilizando el Modelo de Creencias en Salud, una de las teorías más aceptadas para personalizar mensajes de salud pública, estudios con niños han reportado ser útiles para promover la frecuencia de cepillado, uso de seda dental y conductas de higiene. $^{(1)}$

Para motivar el cepillado dental en niños se deben entregar mensajes positivos, destacando los beneficios obtenidos con una buena higiene oral, tal como una buena salud general, autoimagen y especialmente buena apariencia. Si bien la apariencia no es lo más importante, es algo que pueden ver y por lo tanto se puede razonar con ellos, motivándolos a mantener los dientes "blancos y bonitos". Dentro de los beneficios entregados en los mensajes, se sugiere incluir la relación entre mala higiene oral con ataques al corazón, la relación entre fumar y cáncer oral, y el hecho de agradar/ayudar a sus padres, destacando lo efectivo y fácil del cepillado. Si bien todos los escolares creían poder realizar un buen cepillado, se olvidaban con frecuencia, o no lo realizaban por falta de tiempo, cansancio, porque no es una actividad agradable o por estar fuera de su hogar. Los escolares respondieron de forma positiva al sugerir el uso del computador para enviar recordatorios para cepillarse los dientes, siendo un cambio al recordatorio diario "odiado" de sus padres. ${ }^{\left({ }^{8}\right)}$

Estudios indican que ocurren cambios en el proceso de decisiones para adquirir un hábito entre el inicio y la mantención de éste. Se inicia un hábito por miedo a las consecuencias, pero se mantiene por la satisfacción de los resultados. Este punto es clave ya que se deberá diferenciar el mensaje personalizado enviado a aquellos niños sin el hábito de cepillado dental, de aquellos que sólo necesitan reforzarlo. ${ }^{(1,8)}$ 
Si bien existen numerosos estudios de mHealth, no existe mucha evidencia acerca de Apps para la salud oral, menos en niños. Tiffany et al (2018) revisaron 33 Apps de salud oral en inglés, disponibles en App Store y Google Play. Encontraron que dos tercios de las Apps eran dirigidas al público general, la mayoría con descarga gratis y sólo 19 de las 33 Apps utilizaban mensajes de encuadre positivo y/o negativo para influenciar el comportamiento respecto a la salud oral. Todas las Apps deberían enfocarse en mensajes de encuadre positivo ya que se ha demostrado su efectividad para mejorar la salud oral. La mayoría de las Apps evaluadas no presentaban fundamento teórico para los contenidos, ni habían realizado estudios para validación empírica. Además, se encontró que pocas incluían información acerca de la relación entre la dieta, tabaco y alcohol como factores de riesgo para la salud oral. ${ }^{(5)}$

Parker et al. (2019) encontraron 1,075 Apps disponibles en AppStore y/o Google Play relacionadas con higiene oral. Se evaluaron las 20 apps más populares, las preferidas eran aquellas gratis y que incluían más de una característica de gamification, educación de higiene oral y temporizador para el cepillado dental. Respecto al contenido, las apps evaluadas no presentaban base teórica aprobada por organizaciones de salud dental, ni estudios de prueba. Tampoco se mencionaba si se basaron en una teoría de cambio comportamientos, lo que es fundamental para que sean más efectivas. ${ }^{(19)}$

Las Apps que han aumentado con gran rapidez son aquellas dirigidas a pacientes en tratamiento de ortodoncia, con el objetivo de mejorar el compromiso de los pacientes respecto a su higiene bucal, citas con el ortodoncista y cuidados con los frenillos. $(17,19,29)$ Sharif et al. encuestaron a pacientes ortodóncicos entre 8-52 años (9 menores de 11 años, 71 pacientes entre 11 y 18 años, 20 mayores de 18 años), de los cuales el $90 \%$ tenía acceso a un smartphone. El 87\% de los pacientes encuestados indicaron que utilizarían una app para pacientes en tratamiento ortodóncico si existiera, pero sólo 7\% sabía de la existencia de las múltiples apps disponibles, y tampoco habían utilizado una. Si bien existen estudios que han reportado buenos resultados con apps para pacientes ortodóncicos, se necesita evaluar la calidad de las apps disponibles actualmente. (17) Zotti et al. realizaron un estudio en 80 adolescentes (promedio 14.1 años en los 40 adolescentes del grupo intervenido, 13.6 años en promedio del grupo control) que iniciaron tratamiento ortodóncico con brackets. Con el uso de una App de chat anónimo grupal, se buscó mejorar su higiene oral mediante la participación activa con el envío de selfies de sus dientes antes y después de utilizar revelador de placa, y un ranking semanal de los mejores pacientes. La higiene oral mejoró en los pacientes intervenidos respecto al grupo control, disminuyendo el índice de placa, índice gingival y cantidad de manchas blancas a los 6, 9 y 12 meses desde el inicio del estudio. El uso de la App permitió a los pacientes involucrarse directamente y motivarse en mantener su salud oral sin la necesidad de los padres, por lo que es un método que puede utilizarse para mejorar el compromiso de los pacientes en odontología. ${ }^{(29)}$

Por otro lado, Kay y Shou (2019) probaron una App móvil asociada a un dispositivo con sensor de movimiento en el cepillo dental para mejorar la higiene oral en adultos. El grupo con la App disminuyó un $70 \%$ la cantidad de placa bacteriana total, mientras que el grupo control con instrucciones de higiene oral redujo la placa un $30 \%$. Los pacientes reportaron gran aceptación con la App y el dispositivo, además de transformar el cepillado dental en una "experiencia divertida e interesante" y poder "cepillarse por 2 minutos sin saberlo". ${ }^{(7)}$ Nolen et al. desarrollaron otro prototipo de App - Toothsense - dirigida a padres y cuidadores de niños menores de 6 años en Estados Unidos, basados en la Teoría del Comportamiento Planeado, con el objetivo de mejorar hábitos de salud oral en niños. Los resultados demostraron que es efectivo el uso de ésta teoría como base en App para mejorar la salud oral. ${ }^{(2)}$

Campos et al., desarrollaron la App "1,2,3...Brush!" para preescolares de 3-5 años, probada en 43 preescolares de un colegio público de Brasil. Si bien los niños de 3 años requirieron asistencia para utilizar la App, el juego educativo obtuvo resultados positivos en los aspectos de efectividad, satisfacción de usuarios y eficiencia, demostrando la capacidad de los preescolares de utilizar correctamente la App dirigida a ellos. ${ }^{(3)}$

BrushDJ es una App disponible para descargar gratis que presenta evidencia científica para motivar la higiene oral que entrega información de higiene oral, toma el tiempo de cepillado entregando recordatorios de cepillar todos los dientes, utilizar hilo dental, entre otros y envía notificaciones al dispositivo móvil para recordar cepillarse los dientes. Otras propiedades de la App son la posibilidad de compartir las canciones escuchadas en redes sociales, 
y recibir aplausos y una sonrisa al terminar los dos minutos de cepillado. Underwood et al. aplicaron una encuesta voluntaria a los usuarios de BrushDJ, obteniendo respuesta de 189 (131 género femenino), de los cuales 21 tenían 13-17 años, 69 tenían 7-12 años, y 9 usuarios eran menores de 7 años; el resto era mayor a 18 años. El 80\% respondió que la App los motivaba a cepillar sus dientes por más tiempo y un $92,3 \%$ la recomendaría. El $70 \%$ aseveró sentir sus dientes más limpios desde que comenzó a utilizar la App BrushDJ y 39,3\% que sus encías sangraban menos. Cuando se preguntó por qué la App los ayudaba a cepillarse los dientes, la respuesta principal fue motivación (para cepillarse los dientes, cepillarse por más tiempo, entretenerse cepillándose los dientes con música y que ya no ser una tarea aburrida), seguido por educación (les ayudaba a seguir el orden correcto para higienizar sus dientes y a cepillar todos los dientes, no sólo los más visibles; los videos también ayudaban), luego compromiso (mantener la rutina, recordar cepillarse los dientes y mantener el cepillado por 2 minutos) y finalmente beneficios percibidos por los usuarios o hijos de los usuarios (saber que están realizando una correcta higiene oral que mantendrá la salud oral, los dientes blancos y ayudará a no tener caries).(16)

\section{DISCUSIÓN}

Mediante mHealth es posible educar a la población y realizar promoción de la salud bucal. Las Apps de salud oral constituyen un medio importante para acceder a cuidados e información de salud oral, creando un nuevo ambiente de aprendizaje para disminuir inequidades en salud y entregar información para la promoción de la salud oral. ${ }^{(15)}$

El conocimiento es indispensable para generar cambios de conducta y actitud, pero no se asegura el éxito con la sola entrega de información. $(9,10)$ Está comprobado que creer en la habilidad de realizar una actividad por sí mismo predice cómo resulta, lo que es importante para el comportamiento en salud oral. Mientras más retroalimentación positiva se reciba, más probable es que se realice bien la actividad debido a la sensación de logro y dominio de ésta. ${ }^{(7)}$ Para que los niños mantengan buenos hábitos de higiene oral, es necesario motivarlos además de educarlos para mantener el hábito a largo plazo y cambiar su conducta. ${ }^{(30)}$ Combinar los métodos práctico y motivacional, incluyendo la colaboración de pro- fesionales de salud, personal de establecimientos educacionales y padres, permitirá obtener mayores beneficios para la salud oral que se mantengan en el tiempo. ${ }^{(6)}$

Las Apps de higiene oral actualmente disponibles son múltiples, pero no cuentan con información revisada por organizaciones de salud, por lo que pueden contener errores. ${ }^{(23)}$ Por ello es necesario establecer parámetros comunes para estandarizar los futuros estudios y Apps. ${ }^{(21)}$ Stoyanov et al. crearon y validaron las escalas MARS y uMARS para evaluar la calidad de las Apps mHealth y servir de guía para la creación de otras nuevas, incorporando los ítems de estética, información entregada, funcionalidad y compromiso para la evaluación, de manera objetiva. La escala MARS es confiable mientras sea aplicada por profesionales entrenados, por ello se simplificó la escala a uMARS, la cual puede ser aplicada por los mismos usuarios de cada App. ${ }^{(31,32)}$ Además, uMARS entrega la posibilidad de obtener retroalimentación de los usuarios que aplican la escala, contribuyendo a mejorar la calidad de la App. ${ }^{(32)}$

Estudios cualitativos con entrevistas a la población de interés ayudará a mejorar la calidad de las Apps, preguntando acerca de lo que a ellos les gustaría incorporar. Antes de recomendar Apps, los dentistas deberían comprobar la información entregada por la App, además de su calidad. Recientemente, NHS comenzó a evaluar Apps que estén respaldadas con estudios científicos con la iniciativa de recomendarlas a los pacientes. ${ }^{(19)}$

\section{CONCLUSIÓN}

La tecnología con smartphones ha crecido a un ritmo acelerado junto con el desarrollo de Apps, sin embargo, la investigación no ha llevado el ritmo. Se necesitan estudios estandarizados y específicos para cada población, especialmente en niños, antes de aprobar una App y dejarla disponible para descargar. De esta manera se podrán generar cambios efectivos a largo plazo respecto a los hábitos de salud oral de niños y adultos.

\section{BIBLIOGRAFIA}

1. Walker K, Jackson R. The Health Belief Model and Determinants of Oral Hygiene Practices 
and Beliefs in Preteen Children: A Pilot Study. Pediatr Dent 2015;37(1):40-45.

2. Nolen SL, Giblin-Scanlon LJ, Boyd LD, Rainchuso L. Development and Testing of a Smartphone Application Prototype for Oral Health Promotion. J Dent Hyg 2018 Apr;92(2):6-14.

3. Campos LFXA, Cavalcante JP, Machado DP, Marçal E. Development and Evaluation of a Mobile Oral Health Application for Preschoolers. Telemed J E Health 2018 Jul; http://doi. org/10.1089/tmj.2018.0034.

4. Scheerman JFM, Van Empelen P, Van Loveren C, Van Meijel B. A Mobile App (WhiteTeeth) to Promote Good Oral Health Behavior Among Dutch Adolescents with Fixed Orthodontic Appliances: Intervention Mapping Approach. JMIR Mhealth Uhealth 2018;17(6):e163.

5. Tiffany B, Blasi P, Catz SL, McClure JB. Mobile Apps for Oral Health Promotion: Content Review and Heuristic Usability Analysis. JMIR Mhealth Uhealth 2018;6(9):e11432.

6. Markeviciute G, Narbutaite J. Effectiveness of a Motivation and Practical Skills Development Methods on the Oral Hygiene of Orphans Children in Kaunas, Lithuania. J Oral Maxillofac Res 2015;6(3):e2.

7. Kay E, Shou L. A randomised controlled trial of a smartphone application for improving oral hygiene. Br Dent J 2019;226(7):508:511.

8. Walker KK, Steinfort EL, Keyler MJ. Cues to Action as Motivators for Children's Brushing. Health Commun 2015;30:9, 911-921.

9. Aljafari A, Gallagher JE, Hosey MT. Can oral health education be delivered to high-caries-risk children and their parents using a computer game? - A randomised controlled trial. Int J Paediatr Dent 2017;27:476-485.

10. Habbu SG, Krishnappa P. Effectiveness of oral health education in children - a systematic review of current evidence (2005-2011). Int Dent J 2015;65:57-64.

11. Aljafari A, Rice C, Gallagher JE, Hosey MT. An oral health education video game for high caries risk children: study protocol for a randomized controlled trial. Trials 2015;16:237.

12. Anderson-Lewis C, Darville G, Mercado RE, Howell S, Di Maggio S. mHealth Technology Use and Implications in Historically Underserved and Minority Populations in the United States: Systematic Literature Review. JMIR Mhealth Uhealth 2018;6(6):e128.

13. Toniazzo MP, Nodari D, Muniz FWMG, Weidlich P. Effect of mHealth in improving oral hygiene: a systematic review with meta-analysis. J Clin Periodontol 2019 Mar;46(3):297-309.

14. Lee $M$, Lee $H$, Kim $Y$, Kim H, Cho M, Jang $\mathrm{J}$, et al. Mobile App-Based Health Promotion Programs: A Systematic Review of the Literature. Int $\mathrm{J}$ Environ Res Public Health 2018;15:e2838.

15. Faria IM, Sancas MC, Pintor AVB, Primo LS. Dental Apps for Smartphones: New Way of Providing Services and Education. CE 2018;9:687-696.

16. Underwood B, Bridsall J, Kay E. The use of a mobile app to motivate evidence-based oral hygiene behaviour. Br Dent J 2015;219(4):E2.

17. Sharif MO, Siddiqui NR, Hodges SJ. Patient awareness of orthodontic mobile phone apps. J Orthod 2019;46(1):51-55.

18. Stephan LS, Almeida ED, Guimaraes RB, Ley AG, Mathias RG, Assis MV, et al. Processes and Recommendations for Creating mHealth Apps for Low-Income Populations. JMIR Mhealth Uhealth 2017;5(4):e41.

19. Parker K, Bharmal PZ, Sharif MO. The availability and characteristics of patient-focused oral hygiene apps. Br Dent J 2019;226(8):600604.

20. Mendiola M, Kalnicki M, Lindenauer S. Valuable Features in Mobile Health Apps for Patients and Consumers: Content Analysis of Apps and User Ratings. JMIR Mhealth Uhealth 2015;3(2):e40.

21. Bradway M, Carrion C, Vallespin B, Saadatfard O, Puigdomenech E, Espallargues M, Kotzeva 
A. mHealth Assessment: Conceptualization of a Global Framework. JMIR Mhealth Uhealth 2017;5(5):e60.

22. Schoeppe S, Alley S, Van Lippevelde W, Bray NA, Williams SL, Duncan MJ, et al. Efficacy of interventions that use apps to improve diet, physical activity and sedentary behaviour: a systematic review. Int $\mathrm{J}$ Behav Nutr Phys Act 2016;13(1):127.

23. Orji R, Moffat K. Persuasive technology for health and wellness: State-of-the-art and emerging trends. Health Informatics $\mathrm{J}$ 2016;24(1):66-91.

24. Davis TL, DiClemente R, Prietula M. Taking mHealth Forward: Examining the Core Characteristics. JMIR Mhealth Uhealth 2016;4(3):e97.

25. Brick C, McCully SN, Updegraff JA, Ehret PJ, Areguin MA, Sherman DK. Impact of Cultural Exposure and Message Framing on Oral Health Behavior: Exploring the Role of Message Memory. Med Decis Making 2016;36(7):834843.

26. Sardi L, Idri A, Fernández-Alemán JL. A Systematic Review of Gamification in e-Health. J Biomed Inform 2017 Jul;71:31-48.

27. Sailer M, Hense JU, Mayr SK, Mandl H. How gamification motivates: An experimental study of the effects of specific game design elements on psychological need satisfaction.
Comput Human Behav 2017;69:371-380.

28. Maffetone PB, Laursen PB. Decision-Making in Health and Fitness. Front Public Health 2019 Jan; 7:6.

29. Zotti F, Dalessandri D, Salgarello S, Piancino M, Bonetti S, Visconti L, et al. Usefulness of an app in improving oral hygiene compliance in adolescent orthodontic patients. Angle Orthod 2016;86(1):101-107.

30. Teixeira AFB, Kuhn E, Bordin D, Kozlowski VA, Raggio DP, Fadel CB. Infant motivation in dental health: Attitude without constant reinforcement. J Indian Soc Pedod Prev Dent 2014;32(3):225-230.

31. Stoyanov SR, Hides L, Kavanagh D, Zelenko O, Tjondronegoro D, Mani M. Mobile App Rating Scale: A New Tool for Assessing the Quality of Health Mobile Apps. JMIR Mhealth Uhealth 2015;3(1):e27.

32. Stoyanov SR, Hides L, Kavanagh DJ, Wilson $H$. Development and Validation of the User Version of the Mobile Application Rating Scale (uMARS). JMIR Mhealth Uhealth 2016;4(2):e72.

\section{AUTOR DE CORRESPONDENCIA:}

Ricardo Cartes-Velásquez.

Beltrán Mathieu 7, Concepción, Chile.

Fono: 56977575655.

Email: cartesvelasquez@gmail.com 